\title{
Understanding the stoichiometric diversity and reactions of cocrystals through experimental and computational studies
}

\author{
F Topic ${ }^{1}$, M Arhangelskis ${ }^{2}$, P Hindle ${ }^{1}$, R Tran $^{3}$, A Morris $^{3}$, T Friscic ${ }^{1}$ \\ ${ }^{1}$ Mc Gill University, Montreal, Quebec, ${ }^{2}$ University of Warsaw, Warsaw, Poland, ${ }^{3}$ University of \\ Birmingham, Birmingham, United Kingdom \\ filip.topic@mcgill.ca
}

\begin{abstract}
Halogen bonding, a weak interaction formed between the electropositive areas of halogen atoms as donors and electron rich atoms and moieties as acceptors, has in the last two decades been established as a useful addition to the toolbox of supramolecular interactions, conceptually similar yet very different from hydrogen bonding. During this time, solid-state has emerged as the critical medium for the studies of halogen bonding, with crystallography as the most important tool. Cocrystallization in particular has enabled easy access to a large number of systems where the influence of a range of donors and acceptors on the halogen bond properties can be studied. Using di- or multi-topic halogen bond donors and acceptors opens the possibility of obtaining cocrystals comprising same components in different stoichiometric ratios - [i]stoichiomorphs[/i], as not all donor/acceptor sites will necessarily engage in halogen bonding. Moreover, other interactions, such as weak hydrogen bonds involving $\mathrm{C}-\mathrm{H}$ donors and aromatic ring stacking can also more or less successfully compete with the halogen bonding. Outcomes of such cocrystallization reactions can often be tuned by adjusting the stoichiometric ratio of the coformers. In addition to stoichiomorphism, polymorphism can also complicate the structural landscape of even a very simple halogenbonded cocrystal system.[sup]1[/sup]
\end{abstract}

Mechanochemistry has been demonstrated to be a highly efficient and rapid screening method for the formation of molecular solids such as cocrystals.[sup]2[/sup] Bypassing potential solubility issues,[sup]3[/sup] it allows for easy stoichiometric control, as two or more coformer solids can be directly transformed into the novel cocrystalline form.[sup]2[/sup] Furthermore, interconversion between cocrystals of different stoichiometries can be easily achieved mechanochemically, by adding a small amount of the respective coformer and grinding.[sup]2,4[/sup] Such transformations can also be followed both [i]in situ[ii] and [i]ex situ[/i] by a range of diffraction and spectroscopic techniques.

In our work, we focused on a small set of bromine- or iodine-based halogen bond donors and nitrogen-based acceptors. Their cocrystallization was studied by neat or liquid-assisted grinding, and the milling times and stoichiometric ratios of donors and acceptors were varied. Phase analysis was conducted by powder X-ray diffraction, with the structure analysis performed by single-crystal X-ray diffraction. Finally, state-of-the-art periodic DFT calculations were used to establish the relative stabilities of different phases.[sup]1,4-6[/sup]

We found that the stoichiometry of the cocrystals formed in mechanochemical cocrystallization reactions is reliably determined by the starting stoichiometric ratio of the coformers. However, through ex situ analysis, we observed the formation of different stoichiometric variants and polymorphs as the intermediates during cocrystallization. We also used periodic DFT calculations to predict the possibility of interconversion of different stoichiometric variants, which was subsequently confirmed experimentally.

\author{
References \\ $1 \quad$ F. Topić [i]et al.[/i], [i]Chem. Commun.[/i], 2019, [b]55[/b], 14066-14069. \\ 2 S. Karki, T. Friščić and W. Jones, [i]CrystEngComm[/i], 2009, [b]11[/b], 470-481. \\ 3 T. Friščić [i]et al.[/i], [i]CrystEngComm[/i], 2009, [b]11[/b], 418-426. \\ 4 M. Arhangelskis [i]et al.[/i], [i]Chem. Commun.[/i], DOI: 10.1039/D0CC02935A. \\ $5 \quad$ K. Lisac [i]et al.[/i], [i]Cryst. Growth Des.[/i], 2018, [b]18[/b], 2387-2396. \\ $6 \quad$ K. Lisac [i]et al.[/i], [i]Nat. Commun.[/i], 2019, [b]10[/b], 61.
}

Acta Cryst. (2020). A76, a118 